\title{
Scaling in force spectroscopy of macromolecules
}

\author{
Cristiano L. Dias, ${ }^{1}$ Martin Dubé, ${ }^{2}$ Fernando A. Oliveira, ${ }^{3}$ and Martin Grant ${ }^{1}$ \\ ${ }^{1}$ Physics Department, Rutherford Building, McGill University, 3600 Rue University, Montréal, Québec, H3A 2T8 Canada \\ ${ }^{2}$ CIPP, Université du Québec à Trois-Rivières, C.P. 500, Trois-Rivières, Québec, G9A 5H7 Canada \\ ${ }^{3}$ Institute of Physics and ICCMP, Universidade de Brasilia, Campus Darcy Ribeiro, Caixa Postal 04513, \\ 70919-970 Brasilia DF, Brazil
}

(Received 19 December 2004; published 28 July 2005)

\begin{abstract}
We use molecular dynamics to determine the force needed to rupture a chain molecule being stretched at constant loading rate and temperature. When all energy bonds of the molecule are identical, we find that the force $F$ depends on the pulling rate $r$ and temperature $T$ according to $F \sim$ const $-T^{1 / 3}|\ln (r / T)|^{1 / 3}$. When a single weak bond is introduced, this result is modified to $F \sim$ const $-T^{2 / 3}|\ln (r / T)|^{2 / 3}$. This scaling, which is model independent, can be used with experiment to quantitatively extract relevant microscopic parameters.
\end{abstract}

DOI: 10.1103/PhysRevE.72.011918

\section{INTRODUCTION}

Many essential biological processes for life depend on the reaction of various bonds and/or molecules to an applied force. One such example is how Leukocytes recognize invading pathogenic organisms in blood vessels [1]. Atomic force microscope (AFM) [2,3] and biomembrane force probe [4-6] (BFP) are now being used to determine the energy landscape of these complex molecules. In the former experiment, a molecule is attached to the tip of the AFM while a counter-molecule is held at the surface. A specific bond [7] between these two molecules is formed when the tip is brought close to the surface. By removing the tip from the surface at a constant loading rate and recording the most likely force at which the specific bond breaks, a complete spectrum of force versus loading rate is obtained.

Experimentally, the force is seen to increase approximately with the logarithm of velocity for about 3 or 4 decades. This behavior can be explained in terms of a minimal model [8] in which specific bonds are modeled by a Lennard-Jones potential while the surrounding environment of the bond is described by a stochastic force. The breaking of the bond then takes place through thermal fluctuations, which depends strongly on the energy barrier [9]. The potential energy of the bond added to the energy associated with the external force (the energy of the cantilever) accounts for the energy landscape [10]. An approximate solution of the model reveals that the energy barrier of the landscape decreases linearly with the applied force. This linear dependence then results in a linear force spectrum.

There is a need to expand the minimal model and to perform a detailed theoretical study of the breakup process. In particular, it was recently shown that the linear dependence of the energy barrier on the applied force is rarely valid in similar cases where an interplay between thermal fluctuations and a time-dependent energy barrier exists [11-13]. The linear logarithmic behavior must be replaced by a $\ln ^{2 / 3} T / r$ dependence, where $T$ is temperature and $r$ is the loading rate. Since this behavior is observed in systems as varied as Josephson junctions [13], friction of an AFM tip on a surface in the creep regime $[12,14]$, and possibly spin glasses [15], it raises the question of the universality of this result and of its applicability to the breaking of biological molecules.
PACS number(s): 87.15.Aa, 82.37.Rs, 89.75.Da, 05.40.-a

We first use molecular dynamics to study the breaking rate of chain molecule held at a fixed length. We find that the breakup is a thermally activated process and extract the energy barrier and attempt frequency. Two situations are examined. In the first, the chain is composed of identical LennardJones atoms with periodic boundary conditions. We find that the energy barrier $\Delta E \sim\left(s_{c}-s\right)^{3}$, where $s$ is the strain of the chain and $s_{c}$ is the critical strain, the strain at which breakup would occur at $T=0$. This differs from the universality classes described above, and arises from specific boundary conditions. A more realistic situation is to consider the chain of atoms attached to an harmonic spring representing the membrane of a BFP setup, or to be composed of links with different forces. In this situation, the energy barrier $\Delta E$ $\sim\left(s_{c}-s\right)^{3 / 2}$, within the ramped creep universality class. A non-linear vanishing of the energy barrier has been observed in other model biological systems [16,17], and a similar scaling form has also been proposed by Dudko et al. [18].

These results are then used to discuss the dynamical breakup. We use a quasistatic approximation, in which the energy barrier $\Delta E(t) \sim\left(s_{c}-s(t)\right)^{\gamma}$. We show that when the tension is increased at a constant rate, the strain $s$ at which the system ruptures scales with the temperature $T$ and the loading rate $r=d s / d t$ as

$$
\left(s-s_{c}\right) \sim T^{1 / \gamma}[\ln (\kappa T / r)]^{1 / \gamma},
$$

where $\gamma=3$ for identical ring molecule and $\gamma=3 / 2$ for BFP experiments. These results are confirmed by a direct molecular dynamics study of breaking on a simplified BFP set-up. Even though the rate of elongation that can be simulated using molecular dynamics is unrealistically high, these results validate the quasistatic approximation. Our results can thus be applied to experimentally useful range of stretching rate. In particular, this scaling is based on physical arguments and is universal, the detailed form of the potential enters only through the numerical constant $\kappa$.

\section{MODEL DESCRIPTION AND SIMULATION}

We model the molecule being stretched by a onedimensional chain of $N$ atoms, described by the position $x_{i}$ $(i=1, N)$. The atoms interact together through first-neighbor 

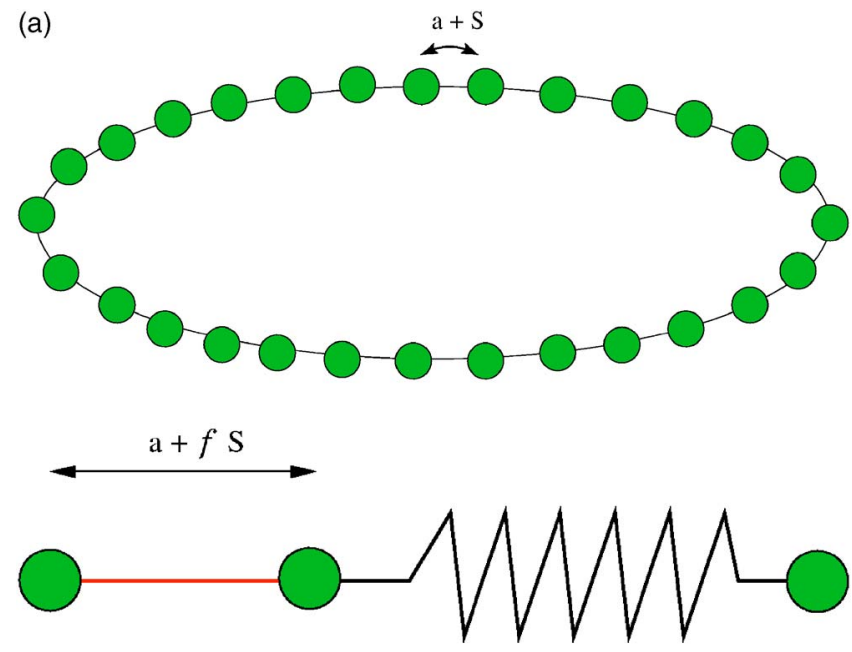

(b)

$$
a+(1-f) S
$$

FIG. 1. (Color online) Schematic representation of stretched chain models. In (a), a periodic chain with similar atoms is presented in its local equilibrium configuration: all atoms stretch by the same amount $S$. In (b) we show a system made by a weak bond and a spring. The spring mimics the membrane of a BFP while the bond models the relevant weak bond of a complex molecule. At metastable equilibrium, a fraction $f$ of the stretched length $S$ of the system extends the weak bond.

interactions only. For specificity, we choose the LennardJones potential (although the exact form of the interaction is not essential)

$$
U(\Delta x)=\epsilon\left[\left(\frac{a}{\Delta x}\right)^{12}-2\left(\frac{a}{\Delta x}\right)^{6}\right]
$$

where $\epsilon$ is the binding energy, $a$ is the interatomic spacing, and $\Delta x$ is the distance between the atoms. The dynamics of this system is obtained by simple Langevin equations. The atoms are immersed in a solvent which acts as a friction force $\left(-\gamma \dot{x}_{i}\right)$ and a random force $\xi_{i}(t)$ on each atom $i$. The intensity of the random force is given by the fluctuationdissipation theorem

$$
\left\langle\xi_{i}(t) \xi_{j}(t+\tau)\right\rangle=\delta_{i, j} \delta(\tau) 2 M \gamma k_{B} T,
$$

where $M$ and $k_{B}$ are the mass and Boltzmann's constant, respectively, and the angular brackets denote an average. The equation of motion of atom $i$ can now be written as

$$
M \frac{d^{2} x_{i}}{d t^{2}}+M \gamma \dot{x}_{i}-F\left(x_{i}-x_{i-1}\right)+F\left(x_{i+1}-x_{i}\right)=\xi_{i}(t),
$$

where $F(x)$ is the force computed from the potential. Two different situations can then be considered.

(1) Periodic chain: The simplest system to study is a periodic chain with $N$ similar atoms, as depicted in Fig. 1(a). Periodic boundary conditions are imposed with the minimum image convention [19] so that the probability of rupture is equal at each point of the chain. The length of the box is fixed at $L=N(a+S)$ and initially all bonds are strained by an equal amount $S$. This setup corresponds to a metastable configuration since the total force on each atom is zero. Therefore, the average strain on each bond stays the same along the simulation until rupture occurs. In that case the broken bond stretches gradually to $a+N S$ while the strain on the others $N-1$ bonds vanishes such that the sum of all bond length is conserved at all times. A similar system, at a fixed length, was already studied by Oliveira [20]. It was found that rupture usually occurred at a single bond through thermal fluctuations.

(2) Attached chain: A more realistic situation is when different bonds (corresponding to different values of $\epsilon$ ) are present in the molecule, with one end of the chain (atom $i$ $=N$ ) strongly attached to a surface and the other (atoms $i$ $=1$ ) to the tip of an AFM or the membrane of BFP. The cantilever of the AFM is modelled by introducing a new atom $x_{0}$, linked to the first atom of the chain by an harmonic spring (with spring constant $k_{h}$ ), leading to an additional term in the potential energy

$$
U_{h}\left(x_{1}-x_{0}\right)=\frac{1}{2} k_{h}\left(x_{1}-x_{0}-a\right)^{2} .
$$

Of particular interest for BFP is when one of the bonds (between atoms $i^{*}$ and $i^{*}+1$ ) is much weaker than the others, and the spring constant less stiffer than the weak bond. Defining $\epsilon(i)$ as the binding energy between atoms $i$ and $i+1$, and representing the weak bond by $\epsilon=\epsilon\left(i^{*}\right)$, the specific situation that we consider is thus

$$
\begin{gathered}
\epsilon(i) / \epsilon \gg 1 \quad \text { for } i=1, i^{*}-1 \\
\epsilon\left(i^{*}\right)=\epsilon \\
\epsilon(i) / \epsilon \gg 1 \text { for } i=i^{*}+1, N-1 .
\end{gathered}
$$

In this case, only the weak bond and the spring plays a role in rupture (the 2 subchains formed by the strong links are essentially undisturbed). Therefore to speed up simulational time we ignore these strong bonds [see Fig. 1(b)]. The atoms at the extremity of the setup are held fixed in place.

Dynamical breakup of the chains: The ultimate goal of a molecular dynamic simulation would be to compute the mean breaking force for chains subjected to strain rates spanning several orders of magnitude (typical experimental time scales range from the millisecond to the minute). However, due to prohibitively long computational times [21], molecular dynamics can only probe breaking for a very short range of loading rates. We thus proceed in two steps, we first calculate the energy barrier for a fixed chain length and show that this result can be used to understand the dynamical breakup. The strain $s$ of the chain is thus fixed (through the boundary conditions) and the time $\tau(s)$ necessary for breakup is obtained.

The dynamics of atoms is given by solving the set of Eq. (4) using the velocity Verlet algorithm $[19,22]$. These are solved numerically for a fixed length of the system (fixed strain) until a bond breaks irreversibly. In order to determine whether rupture has occurred irreversibly, we compute the average time required for the largest bond length of the chain to reach different values $d$. Thermal fluctuations tend to in- 
crease $d$ against the restoring force of the bonds. There is a given value of $d$, which we call $d_{c}$, below which an increase of the largest bond takes a large amount of time. When rupture occurs $\left(\right.$ at $d_{c}$ ) the largest bond increases almost instantaneously since the atoms which are being separated do not resist anymore. In this situation, the resultant force comes from the other bonds which are relaxing to their equilibrium length, thus increasing the length of the largest bond. Within this picture, the average point of rupture $d_{c}$ is well defined.

The mean time of rupture $\tau$ can be computed by knowing the number of chains $N(t)$ that have not ruptured at time $t$. If there are initially $N_{0}$ chains, the time evolution is $N(t)$ $=N_{o} \exp (-t / \tau)$. Unless otherwise stated, we use $N_{o}=800$ in this paper. If breakup is thermally activated, the time for breakup follows an Arrhenius form

$$
\tau(s)=\Omega^{-1}(s) e^{\Delta E(s) / k_{B} T},
$$

where the inverse of the prefactor, $\Omega(s)$, is the attempt breakup frequency and $\Delta E(s)$ is the effective potential energy barrier (i.e., the amount of the energy the heat bath has to supply in order for rupture to occur). The rate of breaking of the chain $R(s)=1 / \tau(s)$.

Simulations are performed in reduced units. Energy is written in terms of $\epsilon$, length is given in terms or $a$ and time is given in units of the smallest period of phonon oscillations $\tau_{o}=2 \pi / 12 \sqrt{\left(2 \epsilon / M a^{2}\right)}$. For simplicity, the mass of each atom is chosen to be one while the friction constant is tuned to $\gamma=0.25\left(2 \pi / \tau_{o}\right)$. Below we present results from simulation. We obtain $\tau(s)$ for various temperatures, from which both the energy barrier $\Delta E(s)$ and the oscillation frequency $\Omega(s)$ can be obtained. The behavior of $\Delta E(s)$ as a function of the strain is particularly important since it appears as an exponential factor in the thermal rate formula [Eq. (7)]. At low strain, we expect linear behavior, i.e., $\Delta E(s) / \Delta E(0)=1+\mathcal{O}(s)$. At some critical strain value $s_{c}$, the energy barrier vanishes and the chain is naturally unstable. Close to $s_{c}$, we expect power-law behavior $\Delta E(s) \sim\left(s_{c}-s\right)^{\gamma}$.

\section{A. Numerical simulations: Attached chain}

This model corresponds to Fig. 1(b). The weak bond is defined by $\epsilon=1$ and $a=1$, such that its stiffness (computed from the Lennard-Jones potential) is $k=72 \epsilon / a^{2}$. The equilibrium length of the harmonic spring is $a$ and its stiffness is $k_{h}=10^{-4} k$. Figure 2(a) shows the dependence of the logarithm of $\tau$ on the inverse of temperature for several values of strain. In agreement with Eq. (7), this dependence is linear, showing that breaking is a thermally activated process, mostly determined by the binding energy $\epsilon$ and the spring constant $k_{h}$. The energy barriers as a function of the strain $s$ are shown in the inset of Fig. 2(a) and demonstrate the existence of a critical value of the strain $s_{c}$. Close to $s_{c}$, the energy barrier scales as $\Delta E \sim\left(s-s_{c}\right)^{\gamma}$, as shown in Fig. 2(b). The best fit yields values of $s_{c}=370 \pm 10$ and $\gamma \approx 1.5 \pm 0.1$; the large uncertainty comes from having to determine two parameters from the fit, together with the constraint that power law behaviors is observed only for strains sufficiently close to the critical strain. The prefactor is given in the inset of Fig.
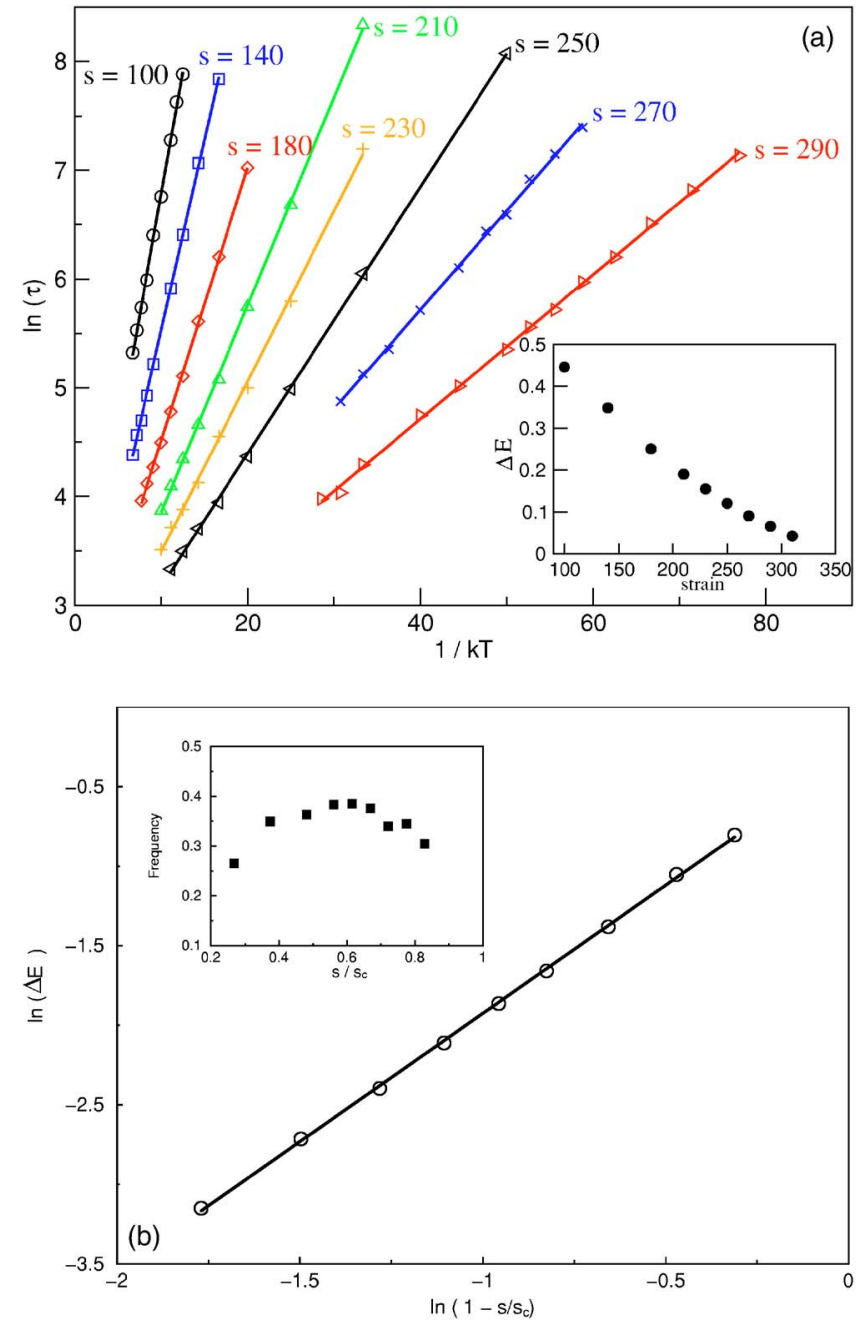

FIG. 2. (Color online) Simulational results for the weak +harmonic chain. In (a) the dependence of breakup time on the temperature and strain is presented. The energy barrier as a function of the strain is shown in the inset. In (b) we show the behavior of the energy barrier and attempt frequency (in the inset) on the strain $s$. The power-law dependence of the barrier is evident.

2(b). It increases with strain until it reaches a maximum and eventually decreases as $s_{c}$ is approached.

\section{B. Numerical simulations: Periodic chain}

In this case, shown in Fig. 1(a), all the bonds of the periodic chains have the same binding energy $\epsilon=1$. Figure 3(a) shows the dependence of the logarithm of $\tau$ on the inverse of temperature for several values of strain and several temperatures. Again, the straight lines shows that breakup is a thermally activated process. The inset of Fig. 3(a) shows $\Delta E(s)$ vs $s$. Again, there exists a critical strain at which the barriers disappears.

We show in Fig. 3(b) that the energy barrier varies as $\Delta E \sim\left(s-s_{c}\right)^{\alpha}$ close to $s_{c}$. A best fit of these simulational data gives $s_{c}=0.115+0.005$ and $\alpha \approx 3.5 \pm 0.1$. The behavior of the prefactor is more complicated. Initially it increases and reaches a maximum as shown in the inset of Fig. 3(b). 

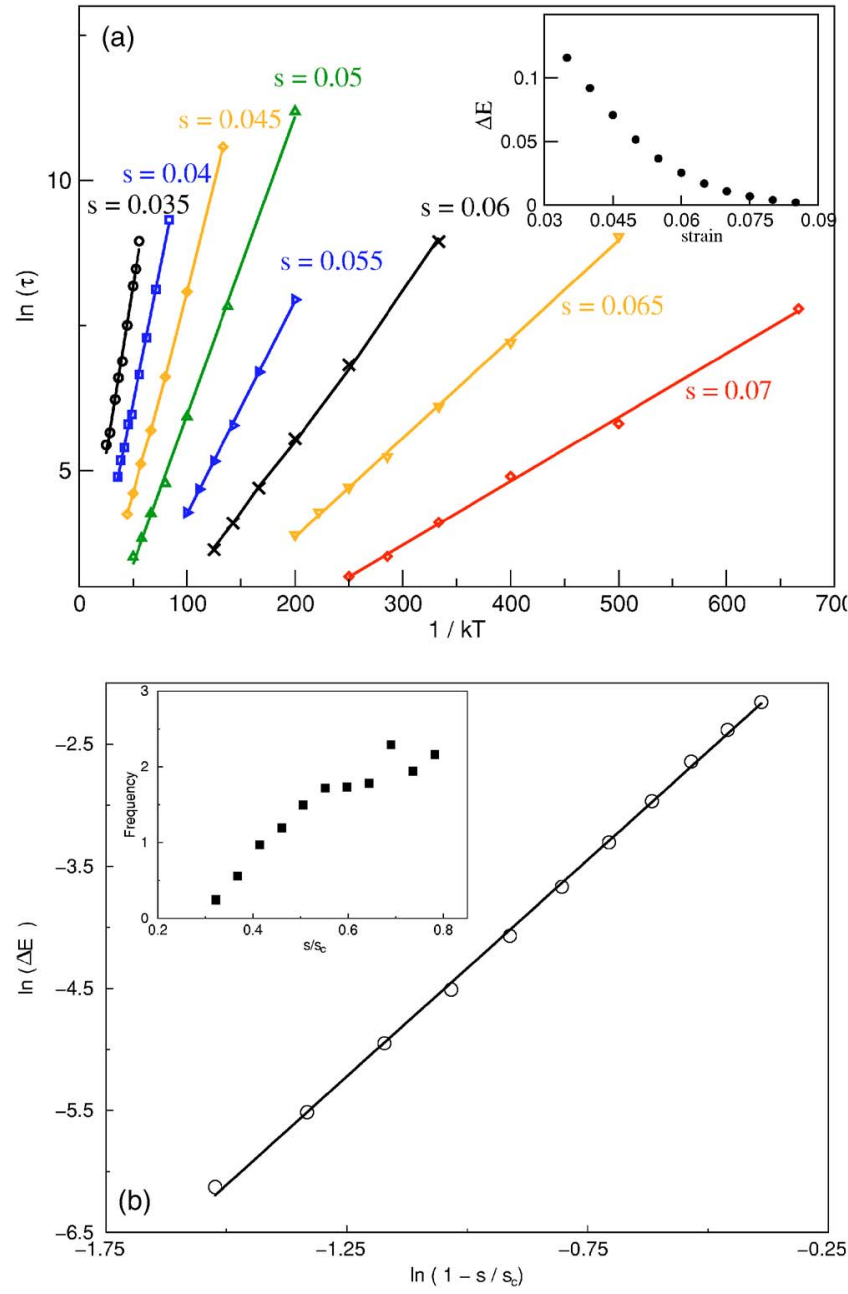

FIG. 3. (Color online) Simulational results for the periodic chain. Different colors correspond to different applied strains. In (a) the dependence of breakup time on temperature and strain is presented. The energy barrier as a function of strain is shown in the inset. In (b) we show the behavior of the energy barrier and attempt frequency (in the inset) on the strain $s$. The power-law dependence of the barrier is evident.

For both the periodic and weak bond chain, the behavior close to $s_{c}$ is very different from the linear dependence usually expected and on which the model of Ref. [8] is built. We now present mean field models that explain these results.

\section{MEAN-FIELD ANALYSIS}

The breakup of a chain can be approached through an effective potential that describes rupture $[20,23,24]$. The potential is built around the idea that the molecule breaks locally at a single specific bond [20]. During breakup, one of the bonds is stretched by an extra amount $\phi$ while the other bonds relax according to the prescribed value of the chain length. The complete breakup process can then be described with the potential $U_{\text {eff }}(s, \phi)$, where $s$ is the prescribed strain of the chain and $\phi$ represents the increase in strain for the breaking bond.
This potential typically has a metastable character. There is one minimum $\phi_{\min }$, located near $\phi=0$ corresponding to a chain which is not broken. This state is separated from a continuum of values of $\phi$ (corresponding to a broken chain) by an energy barrier, with maximum located at $\phi_{\max }$. These points are found by the condition

$$
\left.\frac{\partial U_{\mathrm{eff}}(s, \phi)}{\partial \phi}\right|_{\phi=\phi_{\min }, \phi_{\max }}=0 .
$$

The energy barrier for a given strain $s$ is then

$$
\Delta E(s)=U_{\mathrm{eff}}\left(s, \phi_{\mathrm{max}}\right)-U_{\mathrm{eff}}\left(s, \phi_{\min }\right) .
$$

A zero temperature, the transition to a broken chain occurs when the energy barrier disappear. At this critical strain $s_{c}$, the energy barrier goes to an inflexion point, defined by $\phi_{\min }=\phi_{\max } \equiv \phi_{c}$ and the condition

$$
\frac{\partial^{2} U_{\mathrm{eff}}\left(s_{c}, \phi_{c}\right)}{\partial^{2} \phi}=0 .
$$

At finite temperature, the transition to a broken chain takes place by thermal activation over the energy barrier. The time $\tau$ required for such transition is given by Kramer's reaction rate theory, Eq. (7), with the attempt frequency

$$
\Omega(s)=\frac{\omega_{o}(s) \omega_{b}(s)}{2 \pi \gamma},
$$

where $\omega_{o}$ and $\omega_{b}$ are the vibration frequency around the position of local minima and maximum of energy. Equation (11) is the overdamped approximation of Kramer's result for the attempt frequency [25].

If the load on the chain increases at a constant rate, the energy barrier decreases continuously. Due to the exponential character of the activation rate, breakup will occur for strains $s$ close to the critical strain $s_{c}$, and this even at finite temperature [26]. In the critical region $\left(s_{c}-s\right) / s_{c} \ll 1$, it is possible to obtain easily an accurate approximation for the effective potential, and hence the activation rate. We define $\tilde{s}=1-\left(s / s_{c}\right)$, and expand the effective potential $U(s, \phi)$ in deviations $\delta \phi=\phi-\phi_{c}$ and the small parameter $\widetilde{s}$, from which the energy barrier can thereafter be calculated. Analytic expression for the attempt frequency on $\tilde{s}$ can also be computed by expanding the second derivative to first order on $\widetilde{s}$. We now present the mean-field potential appropriates to both cases.

\section{A. Attached chain}

This case is reprensented in Fig. 1(b). The chain is subjected to the total strain $s$, which is carried by both the weak bond between atoms $i^{*}$ and $i^{*}+1$ and the cantilever between atoms $i^{*}+1$ and the first atom of the setup $i_{o}$. Without fluctuations, the total elongation of the two active bonds is $s$, of which a fraction $f$ is taken by the AFM-chain bond. An approximate estimate of $f$ is obtained by linearizing the Lennard-Jones potential, yielding $f=k_{h} a^{2} / 72 \epsilon$. The path of rupture is parametrized by the quantity $\phi$, describing the extent by which the weak bond length deviates from $a+f s$. 

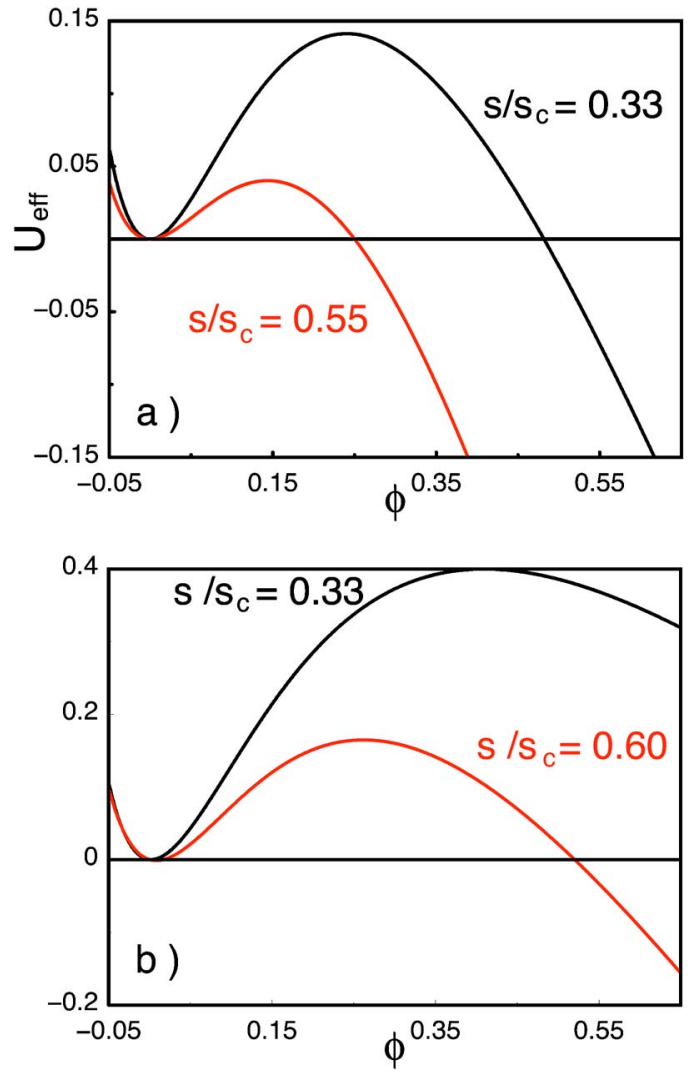

FIG. 4. (Color online) Dependence of the effective energy on $\phi$. Black and red lines correspond to low and high tension respectively. (a) Periodic chain. (b) Chain composed of a weak+harmonic bond.

Equivalently, $\phi$ corresponds to the deviation of the spring from $a+(1-f) s$, where $(1-f) s$ is the strain of the spring in the harmonic approximation. The potential energy along $\phi$ reads

$$
U_{\mathrm{eff}}^{h}(\phi)=\frac{k_{h}}{2}[(1-f) s-\phi]^{2}+U(a+f s+\phi) .
$$

Figure 4 shows how the effective potential for rupture changes when $\phi$ is varied under two different tensions. Low tension is shown in black while high tension is given in red.

Numerical resolutions of Eqs. (8) and (10) show that the critical values $s_{c} / a=373.7$ and $\phi_{c} / a=0.071$. An expansion around these values then leads to an energy barrier

$$
\begin{aligned}
& \Delta E^{h}(\widetilde{s})=E_{0} \widetilde{s}^{3 / 2}, \\
& \Omega^{h}(\widetilde{s})=\Omega_{0} \widetilde{s}^{1 / 2},
\end{aligned}
$$

where $E_{0}$ and $\Omega_{0}$ are given in the footnote [28]. The value of $s_{c}$ and the $\gamma=3 / 2$ exponent for the barrier height are in good agreement with the values obtained by numerical simulation.

\section{B. Periodic chain}

This case is characterized by the constraint of fixed chain length (see Fig. 5) and the energetics of this system is described by the effective potential

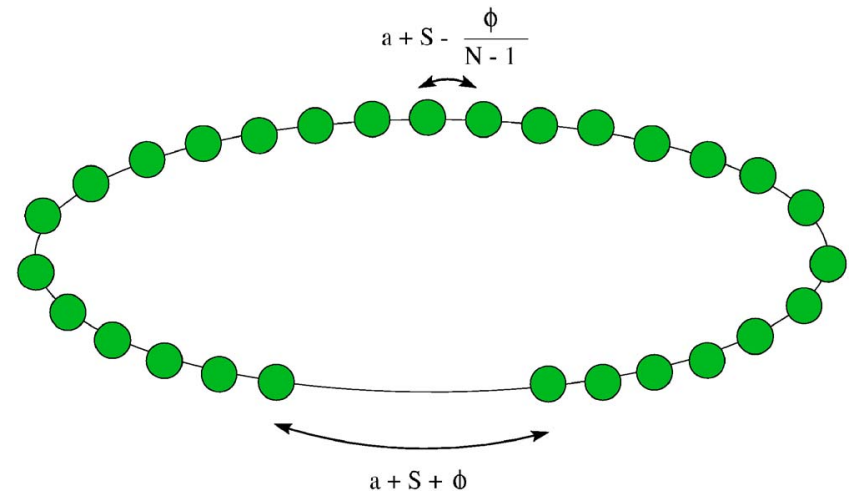

FIG. 5. (Color online) Mean-field description of the breakup of a periodic chain of atoms.

$$
U_{\mathrm{eff}}^{\mathrm{pc}}(\phi)=U(a+s+\phi)+(N-1) U\left(a+s-\frac{\phi}{N-1}\right) .
$$

In this case, $\phi_{\min }=0$ is a minimum for all values of the strain. Since this minimum is fixed, it implies that $\phi_{c}=0$, and the critical strain is simply related to the point at which the single-atom potential becomes unstable

$$
\frac{d^{2} U\left(a+s_{c}\right)}{d s^{2}}=0 .
$$

In the specific case of the Lennard-Jones potential, Eq. (2), this gives $s_{c}=a(13 / 7)^{1 / 6}$.

Due to the particular form of the effective energy of the periodic chain, $\partial^{1+n} U_{\mathrm{eff}}^{\mathrm{pc}} / \partial \phi \partial s^{n}=0$ for all $n$. Therefore higher order Taylor's expansion of Eq. (8) has to be carried out and the resulting scaling for the periodic chain becomes

$$
\begin{aligned}
\Delta E^{\mathrm{pc}}(\widetilde{s}) & =E_{0} \widetilde{s}^{3}, \\
\Omega^{\mathrm{pc}}(\widetilde{s}) & =\Omega_{0} \widetilde{s},
\end{aligned}
$$

where $E_{0}$ and $\Omega_{0}$ can be simply expressed [29]. The exponent in Eq. (16a) is similar to that estimated in the simulation (namely 3.54), and likewise for the value of $s_{c}$.

\section{Crossover scaling}

A crossover scaling function can account for both limits. Consider the parameter $\psi \equiv \partial^{2} U_{\text {eff }} / \partial \widetilde{s} \partial \phi$ which characterizes symmetry: $\psi=0$ represents the periodic (symmetric) chain, while finite $\psi$ corresponds to the assymetric (weak +harmonic) case. Expanding Eqs. (8) and (9) to second order in $\widetilde{s}$ and $\widetilde{\phi}$ gives the scaling form for the energy barrier in the limit of small $\tilde{s}$ and small $\psi$,

$$
E_{b}(\psi, \widetilde{s})=\widetilde{s}^{3} g\left(\psi / \widetilde{s}^{3}\right),
$$

where the scaling function obeys $g\left(\psi^{*} \rightarrow 0\right) \propto 1$, and $g\left(\psi^{*}\right.$ $\rightarrow \infty) \propto\left(\psi^{*}\right)^{1 / 2}$, in terms of the scaled variable $\psi^{*}=\psi / \widetilde{s}^{3}$. The proportionality constants and the form of $g$ are dependent on the derivative of the energy computed at $s_{c}$ and $\phi_{c}$. This recovers the previously obtained symmetric and non- 
symmetric cases in the appropriate limit. Although Eq. (17) is more general than Eqs. (13) and (16), it is preferable to work with these two scalings separately (this is done in the next section).

Notice that by adding a different bond type to the period chain (symmetric case), $\partial^{1+n} U_{\mathrm{eff}}^{\mathrm{pc}} / \partial \phi \partial s^{n} \neq 0$ and we regain the scaling of Eq. (13). In force spectroscopy a different bond (usually harmonic) is required to probe the molecule. Therefore one can say that the scaling of the periodic chain made of equal bonds is not appropriate to describe these experiments.

Finally, we note that Eqs. (13) and (16) are independent on the type of potential used for the interaction between particles.

\section{DYNAMICS OF BREAKING}

The situation of interest for force spectroscopy experiments is when the length of the system is increased at a constant rate $r$ [i.e., $s(t)=r t$ ]. In this case, the chain ruptures at a relative strain $s$ with some probability. Assuming that the breaking of the chain itself takes place on a time scale shorter than any other in the problem, the probability that the chain has not broken at time $t$ is

$$
W(s(t))=\exp \left(-\int_{t_{0}}^{t} \tau^{-1}\left[s\left(t^{\prime}\right)\right] d t^{\prime}\right),
$$

where $\tau(s)$ is Kramer's rate [Eq. (7)] for the static strain $s$. The probability distribution of breakup is simply $P(s)$ $=-d W / d s$ and can be used to obtain the average strain $\langle s\rangle$ at which breakup occurs. This average strain is given [27] by

$$
\langle\widetilde{s}\rangle=\left(\frac{k T}{E_{0}}\right)^{1 / 3} \ln ^{1 / 3}\left[\frac{1}{3}\left(\frac{k T^{2 / 3}}{E_{0}}\right) \frac{s_{c} \Omega_{0}}{r}\right],
$$

for the periodic chain. $E_{0}$ and $\Omega_{0}$ are defined in Eq. (16). For the chain attached to an harmonic spring and containing a single weak bond, it is more relevant to express our results in terms of the force $F$, related to the stain $s$ by

$$
F(s)=k_{h}\left(1-k_{h} a^{2} / 72 \epsilon\right) s .
$$

This is a reasonable approximation for the force since the spring is much softer than the nonlinear bond. If we define $\tilde{f}=1-F / F_{c}$, the probability distribution of rupture force reads

$$
P(\tilde{f})=\Omega^{*} \tilde{f}^{1 / 2} \exp \left[-E_{0}^{* * \tilde{f}^{3 / 2}}-\frac{2}{3} \frac{\Omega^{*} F_{c}}{E_{0}^{*}} e^{-E_{0}^{*} \tilde{f}^{3 / 2}}\right],
$$

where $F_{c} \equiv F\left(s_{c}\right), \Omega^{*} \equiv \Omega_{0} / r, r \equiv d F / d t$, and $E_{0}^{*} \equiv E_{0} / k T$. From this distribution, average breakup is given by [27]

$$
\langle\tilde{f}\rangle=\left(\frac{k T}{E_{0}}\right)^{2 / 3} \ln ^{2 / 3}\left[\frac{2}{3}\left(\frac{k T}{E_{0}}\right) \frac{F_{c} \Omega_{0}}{r}\right],
$$

where $E_{0}$ and $\Omega_{0}$ are defined in Eq. (13).

To derive Eqs. (19) and (22), quasistatic equilibrium was assumed: the mean time of rupture was assumed to obey Kramer's equation at any time. This is justified [21] since experimentally a significant increase in the length of the chain occurs at a macroscopic time scale (determined by experiments and ranging from milliseconds to minutes) which is much larger than the correlation time of molecules in a liquid $\left(\sim 10^{-9} \mathrm{~s}\right)$. Therefore, at any elongation of the chain, the molecule vibrates several times ensuring equilibrium.

Now we present additional simulations relating the scaling given in Eq. (22) to a typical BFP setup. Here, the length of the chain composed of weak + harmonic bond is increased at a constant velocity $v$ : the last atom of the chain is kept fixed while the position of the atom representing the tip of the AFM [see Fig. 1(b)] is given by $x_{0}(t)=x_{0}(0)+v t$. The dynamics is determined by Newton's Law until the chain ruptures. A typical simulational result is presented in Fig. 6(a). Here the force on the spring is shown while the molecule is being stretched at $v=2.7 \times 10^{-3}$ (in units of $a / \tau_{o}$ ) and $T=0.02$ (in units of $\epsilon$ ). The force increases until it drops to zero, indicating rupture. The cusp occurring right at rupture is a typical experimental results of AFM pulling experiments [17] or other stick-slip phenomena $[12,14]$. Note that it is not due to any kind of singulatity in the interatomic potentials but only to the fact that rupture itself is a very fast process compared to the elongation of the chain.

The distribution of breakup force is illustrated in Fig. 6(b) for an ensemble of 1900 chains. These simulations were carried out at $T=0.02$ and two different velocities $v=0.01$ (squares, shown in red) and $v=0.08$ (circles, blue). Lines in this figure correspond to the analytic result [Eq. (21)] and symbols are results obtained from simulation. A good match between simulations and analytical results is observed. We see that the mean force $\langle\tilde{f}\rangle$ of rupture depends on $v$.

To illustrate this dependence, we perform some simulations using ensembles of 1000 chains at three temperatures and various velocities. The mean force $\langle\tilde{f}\rangle$ of rupture is extracted from these simulations. We note that Eq. (22) provides a temperature independent scaling by plotting $\langle\widetilde{f}\rangle^{3 / 2} / T$ in terms of $\ln (T / v)$. Within this choice of axes, data computed at different temperatures collapses into a single line, as shown in Fig. 6(c). The quality of the collapse in this figure for the wide range of temperatures and velocities used in the simulation validates the purposed scaling.

In the simulation performed at the slowest stretching rate [the point at the left-hand side of Fig. 6(b)], each atom of the chain executed on average $\sim 3.3 \times 10^{7}$ oscillations around its equilibrium position before the chain ruptured. If we consider that each vibration is executed in $10^{-9} \mathrm{~s}$ (the vibration period of a molecule in a liquid), than this simulation lasted approximately $33 \mathrm{~ms}$, which is within the time range of experiments. At the other end of the spectrum, only 640 vibrations (or $0.64 \mu \mathrm{s}$ ) were executed before rupture. While this is outside the range of experiments, it is interesting to note that quasistatic approximation remains valid at such a high loading rate.

\section{DISCUSSION AND CONCLUSION}

The typical bond used for force spectroscopy experiments is the specific bond formed between a biotin and a streptavi- 

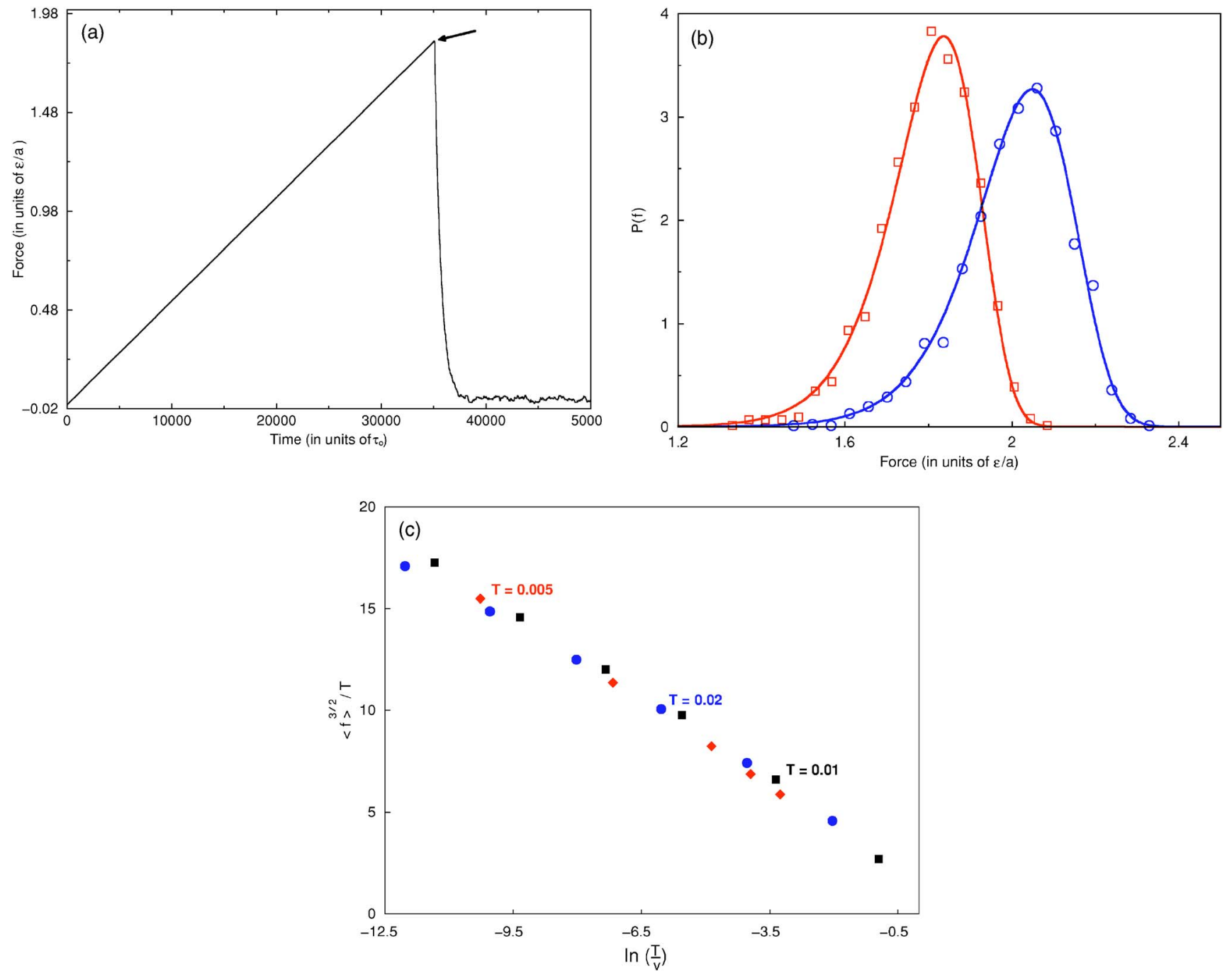

FIG. 6. (Color online) (a) Typical force on the spring while the molecule is being stretched at $v=2.7 \times 10^{-3}$ (in units of $a / \tau_{o}$ ) and $T$ $=0.02$ (in units of $\epsilon$ ). The arrow indicates rupture. (b) (Color online) Normalized probability distribution of breaking force. Simulational results are indicated by symbols. These simulations were performed at $T=0.02$ and $v=0.01$ (squares, red), $v=0.08$ (circles, blue). Curves correspond to analytical result. (c) (Color online) Validation of the scaling form, Eq. (22).

din molecule [4]. These experiments are usually performed at room temperature $\left(k_{B} T=4.1 \mathrm{pN} \mathrm{nm}^{-1}\right)$ and the spring constant used to characterize the membrane of a BFP lies in the range $0.1-3 \mathrm{pN} \mathrm{nm}^{-1}$. The binding energy of the specific bond is $\epsilon \sim 50 k_{B} T=205 \mathrm{pN} \mathrm{nm}$ and its bond length is assumed to be of the order of nanometer $(a \sim 1 \mathrm{~nm})$. If this bond were Lennard-Jones-like, its stiffness would be $72 \epsilon / a^{2}=14760 \mathrm{pN} \mathrm{nm}^{-1}$. In this manner, the stiffness of the membrane is 10000 times smaller than the stiffness of the specific bond. This set of parameters correspond to the blue circles in Fig. 6 which falls in the ramped creep universality class discussed in this paper. Dudko et al. have also proposed the same scaling for force spectroscopy [18]. In their work, they use Morse potential to perform simulations and validate their scaling [which is equivalent to our Eq. (22)]. This provides additional support for our universality argument: The proposed scaling is independent of bond type.

In summary, we modeled the rupture of a specific bond which is being stretched at a constant rate $r$ and temperature
$T$. We showed that if there is just one type of bond being stretched, the strain at which the molecule ruptures scales as: $s \sim T^{1 / 3}\left[\ln \left(T^{1 / 3} / r\right)\right]^{1 / 3}$; when at least two different bonds are stretched, the molecule ruptures according to: $f \sim$ const $-T^{2 / 3}[\ln (r / T)]^{2 / 3}$. Since in force spectroscopy experiments there are at least two types of bonds being stretched (bonds of the molecule and the spring of the BFP), the last scaling should be used to describe these experiments and to extract the relevant parameters from them. In order to test our results experimentally, it would be valuable to probe rupture in an extended range of temperatures.

\section{ACKNOWLEDGMENTS}

C.L.D. would like to thank Yi Sang and Norberto Majilis for useful discussions. This work was supported by the Natural Sciences and Engineering Research Council of Canada, and le Fonds Québécois de la Recherche sur la Nature et les Technologies. 
[1] R. Merkel, Phys. Rep. 346, 343 (2001).

[2] Y.-S. Lo, Y.-J. Zhu, and T. P. B.Jr., Langmuir 17, 3741 (2001).

[3] O. H. Willemsen, M. M. E. Snel, A. Cambi, J. Greve, B. G. D. Grooth, and C. G. Figdor, Biophys. J. 79, 3267 (2000).

[4] R. Merkel, P. Nassoy, A. Leung, K. Ritchie, and E. Evans, Nature (London) 397, 50 (1999).

[5] E. Evans, A. Leung, D. Hammer, and S. Simon, Proc. Natl. Acad. Sci. U.S.A. 98, 3784 (2001).

[6] E. Evans, Biophys. Chem. 82, 83 (1999).

[7] P. Bongrand, Rep. Prog. Phys. 62, 921 (1999).

[8] E. Evans and R. Merkel, Biophys. J. 72, 1541 (1997).

[9] T. Strunz, K. Oroszlan, I. Schumakovitch, H.-J. Guntherodt, and M. Hegner, Biophys. J. 79, 1206 (2000).

[10] G. I. Bell, Science 200, 618 (1978).

[11] K. Binder, Phys. Rev. A 29, 341 (1984).

[12] Y. Sang, M. Dubé, and M. Grant, Phys. Rev. Lett. 87, 174301 (2001).

[13] T. A. Fulton and L. N. Dunkleberger, Phys. Rev. B 9, 4760 (1974).

[14] E. Gnecco, R. Bennewitz, T. Gyalog, C. Loppacher, M. Bammerlin, E. Meyer, and H.-J. Guntherodt, Phys. Rev. Lett. 84, 1172 (2000).

[15] S. Sills and R. M. Overney, Phys. Rev. Lett. 91, 095501 (2003).

[16] P.-C. Li and D. E. Makarov, J. Chem. Phys. 119, 9260 (2003).

[17] G. Hummer and A. Szabo, Biophys. J. 85, 5 (2003).

[18] O. Dudko, A. Filippov, J. Klafter, and M. Urbakh, Proc. Natl. Acad. Sci. U.S.A. 100, 11378 (2003).

[19] M. P. Allen and D. J. Tildesley, Computer Simulations of Liquids (Clarendon, Oxford, 1990).
[20] F. A. Oliveira and P. L. Taylor, J. Chem. Phys. 101, 10118 (1994).

[21] S. Izrailev, S. Stepaniants, M. Balsera, Y. Oono, and K. Schulten, Biophys. J. 72, 1568 (1997).

[22] W. C. Swope, H. C. Andersen, P. H. Berens, and K. R. Wilson, J. Chem. Phys. 76, 637 (1982).

[23] F. A. Oliveira, Phys. Rev. B 57, 10576 (1998).

[24] R. K. Puthur and K. L. Sebastian, Phys. Rev. B 66, 024304 (2002).

[25] P. Hanggi, P. Talkner, and M. Borkovec, Rev. Mod. Phys. 62, 251 (1990).

[26] J. D. Gunton and M. C. Yalabik, Phys. Rev. B 18, 6199 (1978).

[27] A. Garg, Phys. Rev. B 51, 15592 (1995).

[28] For the harmonic + weak link chain

$$
\begin{gathered}
E_{0}=\frac{4}{3} s_{c}^{3 / 2}\left[\left|2\left(\frac{\partial^{2} U_{\text {eff }}}{\partial s \partial \phi}\right) /\left(\frac{\partial^{3} U_{\text {eff }}}{\partial \phi^{3}}\right)\right|\right]^{1 / 2}\left(\frac{\partial^{2} U_{\text {eff }}}{\partial s \partial \phi}\right), \\
\Omega_{0}=s_{c}^{1 / 2}\left[\left|2\left(\frac{\partial^{2} U_{\text {eff }}}{\partial \phi \partial s}\right) /\left(\frac{\partial^{3} U_{\text {eff }}}{\partial \phi^{3}}\right)\right|\right]^{1 / 2}\left(\frac{\partial^{3} U_{\text {eff }}}{\partial \phi^{3}}\right) / 2 \pi M \gamma
\end{gathered}
$$

and the derivatives are computed at $s_{c}$ and $\phi_{c}$.

[29] For the periodic chain

$E_{0}=\frac{2}{3} s_{c}^{3}\left(\frac{\partial^{3} U_{\text {eff }}}{\partial s \partial \phi^{2}}\right)^{3} /\left(\frac{\partial^{3} U_{\text {eff }}}{\partial \phi^{3}}\right)^{2}, \quad \Omega_{0}=s_{c}\left(\frac{\partial^{3} U}{\partial s \partial \phi^{2}}\right) / 2 \pi M \gamma$

and the derivatives are computed at $s_{c}$ and $\phi_{c}$. 